Loading

The Journal of the Canadian Game Studies Association

\title{
La créativité dans l'industrie du jeu vidéo au Québec
}

Un colosse aux pieds d'argile?

\section{Creativity in Quebec Video Game Industry}

\section{A House of Cards?}

\section{Laureline Chiapello}

Volume 14, Number 23, 2021

Le jeu vidéo au Québec

The Video Game in Quebec

URI: https://id.erudit.org/iderudit/1078729ar

DOI: https://doi.org/10.7202/1078729ar

See table of contents

Publisher(s)

Canadian Game Studies Association

ISSN

1923-2691 (digital)

Explore this journal

Cite this article

Chiapello, L. (2021). La créativité dans l'industrie du jeu vidéo au Québec : un colosse aux pieds d'argile ? Loading, 14(23), 77-99.

https://doi.org/10.7202/1078729ar
Article abstract

The province of Quebec is seen as a center of "creativity" for video games and this concept is regularly used and valued. But the complexity of the socio-cultural phenomena leads us to question the meaning of this concept. What is creativity, who or what is creative? This paper uses a constructivist version the Four P's framework (product, process, people and press) to show the limits of the concept of creativity in the Quebec video game industry, specifically in Montreal. This model allows us to focus on different interrelated dimensions of creativity, from government economic policies to the creation process, including the products created and the people involved. At first glance, the results show a disturbing lack of creativity. But it is rather the lack of theorization and operationalization of the concept in the context of Quebec video games that is problematic. If the province wishes to maintain its status as a leader in this sector, a transdisciplinary research effort must be undertaken.
Copyright (c) Laureline Chiapello, 2021

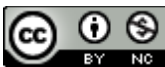

This document is protected by copyright law. Use of the services of Erudit (including reproduction) is subject to its terms and conditions, which can be viewed online.

https://apropos.erudit.org/en/users/policy-on-use/ 
Loading... The Journal of the Canadian Game Studies Association

Vol 14(23): 77-99

http://loading.gamestudies.ca

\title{
La créativité dans l'industrie du jeu vidéo au Québec : un colosse aux pieds d'argile?
}

\section{Creativity in Quebec Video Game Industry: A House of Cards?}

\author{
Laureline Chiapello \\ École des arts numériques, de l'animation et du design (NAD) \\ Université du Québec à Chicoutimi \\ lchiapello@nad.ca
}

\begin{abstract}
Résumé
En matière de jeu vidéo, la province de Québec est vue comme un pôle de "créativité ». La complexité des phénomènes socioculturels nous pousse à nous interroger sur le sens de ce concept souvent invoqué et valorisé. Qu'entend-on par créativité, qui ou qu'est-ce qui est créatif? Cet article s'appuie sur une version constructiviste du modèle des 4 P's (product, process, people, press) pour exposer les limites de la créativité dans l'industrie québécoise du jeu vidéo, en particulier à Montréal. Le modèle adopté ici permet de s'attarder sur différentes dimensions interreliées de la créativité, depuis les politiques économiques gouvernementales jusqu'aux processus de création en passant par les produits créés et les personnes impliquées. Les résultats montrent à première vue une inquiétante absence de créativité. Mais c'est davantage le manque de théorisation et d'opérationnalisation du concept dans le contexte du jeu vidéo québécois qui pose problème. Si l'on souhaite que la province conserve son statut de meneur dans ce secteur, un effort transdisciplinaire de recherche est à entreprendre.
\end{abstract}

\section{Mots-clés}

Jeu vidéo, études de la créativité, transdisciplinarité, modèle socioculturel de la créativité, modèle des $4 \mathrm{P}$, industrie créative, design de jeu, Montréal.

\footnotetext{
Abstract

The province of Quebec is seen as a center of "creativity" for video games and this concept is regularly used and valued. But the complexity of the socio-cultural phenomena leads us to question the meaning of this concept. What is creativity, who or what is creative? This paper uses a constructivist version the Four P's framework (product, process, people and press) to show the limits of the concept of creativity in the Quebec video game industry, specifically in Montreal. This model allows us to focus on different interrelated dimensions of creativity, from government
} 
economic policies to the creation process, including the products created and the people involved. At first glance, the results show a disturbing lack of creativity. But it is rather the lack of theorization and operationalization of the concept in the context of Quebec video games that is problematic. If the province wishes to maintain its status as a leader in this sector, a transdisciplinary research effort must be undertaken.

\section{Keywords}

Video game, creativity studies, transdisciplinarity, sociocultural model of creativity, 4 P's model, creative industries, game design, Montreal.

\section{Introduction}

Le gouvernement provincial n'hésite pas à l'affirmer : «L'industrie québécoise du jeu vidéo jouit d'une réputation internationale enviable. Elle est reconnue pour son innovation, son savoir-faire, sa créativité et, surtout, le succès de ses productions » (Communauté des jeux vidéo du Québec, 2020). L'Alliance numérique a même estimé que la croissance fulgurante de l'industrie vidéoludique québécoise relève du miracle, et que sa force réside dans le talent et la créativité de ses travailleurs (Alliance numérique, 2014). Comme le fait remarquer Robert Paul Weiner dans son histoire de la créativité (Weiner, 2000), quel que soit l'angle sous laquelle elle est abordée, la créativité est depuis le début du $\mathrm{XX}^{\mathrm{e}}$ siècle le plus souvent vue comme une valeur positive, associée à la croissance économique ou au développement de soi. Or, les discours sur la créativité dans l'industrie vidéoludique à Montréal recouvrent des aspects plus discutables.

Ainsi, des studios soutenus par des fonds publics au nom de la créativité connaissent des difficultés majeures. En 2017, Hibernum, un studio indépendant montréalais, a licencié la majorité de ses 115 employés. Ce studio avait été soutenu par le gouvernement du Québec au nom de la créativité : «C'est une entreprise qu'on a appuyée, qu'on a soutenue tout le long, qui était créative, a commenté [...] Chantal Corbeil, porte-parole d'Investissement Québec. C'est vraiment dommage, ce qui arrive » (Benessaieh, 2017). En 2012, THQ et Funcom, deux autres studios soutenus par Investissement Québec, ont aussi subi d'importantes pertes financières. Le premier a déclaré faillite en 2012 (et a finalement été racheté par Ubisoft), et le second a réduit au minimum son nombre d'employés. Paradoxalement, juste avant la vague de licenciements, Funcom avait publié une nouvelle propriété intellectuelle, le jeu en ligne The Secret World. Malgré de bonnes notes des joueurs et des critiques - il a reçu une note moyenne de $74 \%$ (Metacritic, 2012) —, le jeu ne s'est pas vendu suffisamment. En pareil cas, une très forte pression s'exerce sur les directeurs créatifs, et ceux de Funcom ont été rapidement renvoyés. Pourtant, sur la page Metacritic, les critiques de Armchair Empire et de GRY-Online.pl ont souligné que The Secret World était différent, original. Le chef de l'équipe de designers de jeu (lead designer), Martin Bruusgard, a pour sa part estimé que le jeu était trop innovant (Weber, 2012), ce qui semble être un comble : une œuvre trop créative!

Concernant les conditions de travail dans l'industrie, des abus inquiétants sont régulièrement rapportés. En 2004, la pratique consistant à faire travailler les employés beaucoup plus que le temps prévu par la loi, surtout en fin de projet (pratique nommée crunch), a été dénoncée par l'épouse d'un employé d'Electronic Arts sur son blogue, et le billet a été largement relayé. Cela a permis de bien montrer que sous couvert de créativité, des pratiques abusives ont cours dans les 
entreprises : selon leur logique, les emplois créatifs et intéressants vont forcément de pair avec certaines « contreparties », comme des salaires bas et des échéanciers très serrés (Dyer-Witheford et Sharman, 2005).

Ces situations concrètes nous poussent à interroger le concept de créativité : comment est-il défini et utilisé dans le domaine du jeu vidéo ? S'intéresse-t-on à la créativité de l'industrie, des individus, des jeux? Devant la situation ambigüe et complexe du jeu vidéo au Québec, il nous a semblé nécessaire de nous demander quelles en sont les limites. Car c'est autour de ce concept que tournent bon nombre de politiques publiques, de programmes de formation, ou d'occasions de subventions de recherche.

Cet article adopte une approche qualitative sociocritique (Anadón, 2006), et propose une revue de la littérature narrative et transdisciplinaire: "The transdisciplinary literature review is an opportunity to situate the inquirer in an ecology of ideas » (Montuori, 2013, p. 45). Il ne s'agit pas d'effectuer une recension exhaustive et systématique des textes évoquant la créativité dans le domaine du jeu vidéo ou même concernant l'industrie vidéoludique au Québec, mais de s'attarder sur ceux qui permettent d'entrevoir les limites des discours sur la créativité au sein de l'écosystème montréalais. Les textes ont été sélectionnés pour leur position nuancée et pour leur applicabilité au cas du Québec. Sont ainsi privilégiés des rapports québécois et des travaux de chercheurs et chercheuses de la Belle Province.

L'article a pour cadre théorique le modèle socioculturel de la créativité de Vlad Petre Glăveanu. Ce modèle, qui permet de comprendre différentes dimensions de la créativité, sera présenté ciaprès. Prenant acte des perspectives qu'il ouvre, nous exposerons ensuite comment les conceptualisations évoluent et pourquoi elles ne conviennent pas nécessairement pour décrire le secteur du jeu vidéo québécois, ce qui débouche sur une impression générale de manque de créativité. Mais nous verrons que plus qu'un manque de créativité, c'est le manque de théorisation appropriée qui demeure problématique.

\section{Le modèle socioculturel de la créativité : une vision constructiviste des 4 P's}

Dans notre discipline d'attache, le design, le concept de créativité n'est que rarement remis en question ou défini : le design est vu comme un domaine intrinsèquement créatif. Mais cette absence de questionnement montre aujourd'hui ses limites, et il a récemment été souligné qu'une réflexion théorique était nécessaire : "Whatever terms we use to define a designer, a creative designer, or an innovative designer is evidence of a much larger and complex discussion on the relationship between creativity and design » (Hernández, Cooper, Tether et Murphy, 2018, p. 262). On note aussi que dès 1983, l'un des auteurs phares du design, Donald Schön, n'avait pas hésité à écrire que le terme venait « mystifier » les processus de design plutôt que les éclairer (Schön, 1983, p. 187). Ce manque de définition se retrouve dans d'autres disciplines. Néanmoins, rejeter le concept en bloc n'est pas une voie viable, car il est aujourd'hui largement répandu.

C'est pour répondre à cette nécessité définitionnelle que se sont développées les études de la créativité. Au sein même de ce champ de recherche, il est admis que le concept central de créativité est épineux (Glăveanu, 2014; Plucker, Beghetto et Dow, 2004). De plus, si la plupart des ouvrages sur les études de la créativité désignent l'allocution d'investiture de Joy Paul Guilford à la présidence de l'American Psychological Association, en 1950, comme point de départ, on 
reconnait désormais que les études de la créativité sont plus anciennes et présentent une forte hétérogénéité (Glăveanu, 2019).

Dans cet article, nous adhérons à une vision constructiviste et relativement récente de la créativité, que nous allons rapidement situer. En 2014, une nouvelle revue scientifique voyait le jour, Creativity: Theories - Research - Applications (Glăveanu, 2014). Dans le numéro inaugural, Vlad Petre Glăveanu signe un texte provocateur dans lequel il affirme que les études de la créativité sont en crise. Selon lui, elles sont d'une part trop fragmentées, et manquent d'autre part de nouvelles réflexions théoriques. Cela se traduit par la multiplication de projets de faible portée et centrés sur des méthodes de mesure de la créativité, des projets s'appuyant sur des données uniquement quantitatives et menés dans une perspective positiviste. Pour sa part, Creativity: Theories - Research - Applications se veut plus ouverte à la recherche qualitative et à la recherche fondamentale, pluridisciplinaire (la psychologie ayant tendance à accaparer le sujet de la créativité) et internationale. On espère de la sorte créer des liens entre les travaux, unifier et renforcer certaines voies de recherche et distinguer les sujets les plus prometteurs. En 2019, un manifeste publié dans The Journal of Creative Behavior, qu'ont signé vingt chercheurs phares du domaine, prolonge la critique de Glăveanu en défendant l'idée d'un changement durable (Glăveanu et al., 2019). Trop souvent cantonnées à des recherches disciplinaires, quantitatives et fortement marquées par les épistémologies positivistes, les études de la créativité doivent, suivant ces chercheurs, tendre vers une vision plus socioculturelle, appuyée sur des données quantitatives mais aussi qualitatives, et ouverte à différentes épistémologies. Les auteurs relèvent un besoin urgent de manier le concept de créativité en adoptant une attitude critique et réflexive, et de revisiter ce qui a déjà été publié pour mieux l'approfondir et l'enrichir. Cette approche permet aussi de jeter des ponts vers des disciplines dont les orientations épistémologiques sont constructivistes. C'est le cas des disciplines du design, qui, depuis le tournant réflexif amorcé par les travaux de Schön dans les années 1980 (Bousbaci, 2008), ont grandement rejeté la vision positiviste de la pratique. De notre point de vue, les études de la créativité revêtent une pertinence nouvelle grâce à ce repositionnement épistémologique (Chiapello, 2019).

Un signe tangible de ce renouveau au sein des études de la créativité peut être constaté dans la remise en question de certains modèles, comme le 4 P's model de Mel Rhodes. Dans son article "An Analysis of Creativity» (1961), Rhodes définit quatre dimensions nécessaires pour comprendre la créativité : product, people, process et press, termes généralement traduits en français par «produit», " personne », " processus », « environnement». Ces dimensions sont liées à la définition de la créativité de cet auteur : la créativité est le phénomène par lequel une personne élabore (par un processus) et communique un nouveau concept (ou produit) au sein d'un environnement donné.

Au fil du temps, le modèle des $4 \mathrm{P}$ est devenu, selon Mark Runco, « the most often-used structure for creative studies » (Runco, 2004, p. 661). Si la réflexion originale date des années 1960 et relève d'une vision positiviste, et plutôt psychologique et quantitative, de la créativité, elle a été reprise récemment par des auteurs plus constructivistes. Ces auteurs en font valoir la pertinence pour une approche systémique des phénomènes, l'étude d'un phénomène consistant alors à examiner les quatre dimensions ainsi que les rapports qu'elles entretiennent entre elles, et ce, afin de dresser un portrait plus qualitatif de la situation. Cela rend possible la mise en relation des différentes dimensions d'un phénomène socioculturel et permet souvent de dégager une vision nouvelle, 
pluridisciplinaire et critique (Gruszka et Tang, 2017). Le modèle des $4 \mathrm{P}$ est ainsi vu comme un méta-modèle, c'est-à-dire qu'il permet des clarifications conceptuelles d'ordre général plutôt qu'il n'impose une certaine définition de la créativité (Glăveanu, 2013). Il rend explicite la complexité implicite du terme créativité. Souvent abordée sous un seul angle et dans une visée spécifique, ladite créativité recouvre en fait de nombreux phénomènes économiques, sociaux ou culturels. Le rapprochement des quatre aspects mène à de nouvelles vues. Dans notre cas, cela va nous permettre de montrer l'inadéquation entre les conceptualisations et les pratiques observées dans l'industrie québécoise du jeu vidéo.

C'est donc cette nouvelle voie au sein des études de la créativité, dont les travaux de Glăveanu sont représentatifs, que nous empruntons pour cet article. Ce que propose Glăveanu, c'est une interprétation du $4 P^{\prime}$ 's model qui s'éloigne d'une vision individualiste et cognitiviste pour s'approcher d'une vision « écologique » et contextuelle (Glăveanu, 2013). Notons qu'il modifie le nom des dimensions (et en subdivise une) pour arriver au modèle des 5 A's : actor (people), action (process), artifact (product), audience and affordances (press). Nous avons choisi de conserver les termes originaux en $\mathrm{P}$ pour plus de clarté, mais nous adoptons effectivement son approche des dimensions.

Glăveanu souhaite faire émerger l'importance des activités de chacun et éviter un portrait mécaniste et distancié d'un milieu. Tel que le conçoit Glăveanu, le modèle des $4 \mathrm{P}$ nous semble particulièrement pertinent pour la situation du jeu vidéo québécois, qui ne peut être réduite à un seul de ses aspects. Étant nous-même à l'intersection des disciplines du design, des études du jeu et des études de la créativité, nous avons choisi de ne pas nous limiter à une seule discipline, et de rassembler des travaux qui ne communiquent habituellement pas nécessairement entre eux (Montuori, 2013). Cela permet, entre autres, de faire ressortir le manque d'informations sur certains aspects de la conception de jeux vidéo (tout en rappelant cependant que notre objectif n'est pas de donner un portrait complet pour chacune des disciplines). Enfin, Runco (2004) observe aussi qu'étant donné la généralité du modèle, il est nécessaire de l'utiliser dans une visée précise, ce qui est notre cas. En utilisant le cadre des $4 \mathrm{P}$ et les révisions qu'en a proposées Glăveanu, nous souhaitons examiner spécifiquement la situation de l'industrie du jeu vidéo à Montréal.

\section{L’environnement : les industries créatives}

Si la plupart des études recourant au modèle des $4 \mathrm{P}$ se concentrent en premier lieu sur les personnes, cet article souhaite tout d'abord présenter l'environnement global de la créativité vidéoludique au Québec. Dans le modèle d'origine, le contexte est considéré comme un ensemble de variables externes ayant un effet mesurable sur la créativité d'un individu (Glăveanu, 2013). Dans son modèle, Glăveanu souligne plutôt les liens d'interdépendance entre les créateurs et leur environnement aussi bien social que matériel. Dans notre cas, la créativité est actualisée dans les discours par l'idée d'industrie créative (Chambre de commerce du Montréal métropolitain, 2018). Nous verrons dans cette première partie les divers aspects de l'environnement qui sont convoqués pour élaborer le concept d'industrie créative : les faits socioéconomiques, les lois, les crédits d'impôt et même les plans des urbanistes. 


\section{Les industries créatives : une construction politique et économique}

Le concept d'industrie créative est une construction avant tout politique et économique, comme l'affirme le spécialiste des industries culturelles et créatives David Hesmongdale (2008). L'expression a d'ailleurs été popularisée au Royaume-Uni à la fin des années 1990 par le Department of Culture, Media and Sport : «[Creative industries are] activities which have their origin in individual creativity, skill and talent and which have the potential for wealth and job creation through the generation and exploitation of intellectual property » (Ministerial Creative Industries Strategy Group, 2001).

Dans cette vision économique, l'innovation est le moteur de la croissance (Flew, 2002). L'adoption de politiques publiques valorisant ce type d'industries créatives repose sur l'espoir qu'elles produiront de nouvelles propriétés intellectuelles et contribueront à la croissance économique du pays. On remarque un raccourci entre industrie créative et industrie innovante, qui s'appuie sur l'idée que la croissance est aujourd'hui principalement liée à l'innovation :

[E]n moins d'un demi-siècle, le processus de génération des innovations est devenu le terrain de compétition majeur du capitalisme contemporain et un moyen essentiel du développement durable des sociétés contemporaines. Pour les entreprises, nous verrons [...] qu'il s'agit de survivre dans un capitalisme de l'innovation intensive. Dans une société habituée à renouveler régulièrement ses projets et ses modes de vie, il s'agit même du mode fondamental de création de valeur.

Le Masson, Weil et Hatchuel, p. 23 (2006)

On arrive donc à l'idée que s'il y a croissance, c'est parce qu'il y a une forme de créativité.

Cette vision centrée sur l'économie et les entreprises a inspiré différents travaux en créativité organisationnelle partout dans le monde. Montréal n'y échappe pas, où l'on constate l'émergence de recherches qui adhèrent à la vision des industries créatives (Cohendet, Llerena et Simon, 2012; Cohendet et Simon, 2007; Grandadam, Cohendet et Simon, 2012). Hesmondhalgh qualifie ces chercheurs d'accomodationists, car ils acceptent le discours économique dominant :

[...] they broadly accept the position underlying the most influential forms of creative industries policy: that the creative industries are a key new growth sector of economies, both nationally and globally; and that they are therefore the key source of future employment growth and export earnings.

David Hesmondhalgh, p. 562 (2008)

Les chercheurs québécois mettent par exemple l'accent sur une créativité dont l'essor est redevable à un environnement composé de différents acteurs fournissant un cadre social et matériel, et qui a mené à la constitution du pôle montréalais (Grandadam et al., 2012).

Cependant, cet idéal d'un pôle industriel florissant grâce à l'échange et à la mise en commun des connaissances a été remis en question (Drake, 2013; Oakley, 2009; Schlesinger, 2007, 2017; Tremblay, 2008). Tout d'abord, la signification de l'expression industrie créative demeure peu 
claire, surtout au regard du terme industries culturelles préexistant (Hesmondhalgh, 2008), ce que Gaëtan Tremblay, professeur à l'École des médias de l'Université du Québec à Montréal, exprime en ces termes : «Au plan théorique, la notion d'industries créatives n'apporte strictement rien aux travaux sur les industries culturelles. L'on savait déjà pertinemment l'importance qu'y joue la création » (Tremblay, 2008, p. 82). Ensuite, Phillip Schlesinger, chercheur européen connu pour son discours critique, avance que la créativité et les industries créatives sont des concepts érigés en doctrine par certains gouvernements : toute politique part de leur acceptation inconditionnelle, sans questionnement sur leur sens. Pour lui, la notion d'industrie créative témoigne "d'une approche essentiellement économique de la gestion et de la vente de la culture nationale sur le marché mondial » (Schlesinger, 2012, p. 81), et ce, au détriment de la culture elle-même.

\section{Intérêts économiques, crédits d'impôt et créativité}

Cette vision économique de la créativité n'a pas toujours prévalu au Québec. Avec son rapport $A$ Sense of Place, a Sense of Being (Standing Committee on Canadian Heritage, 1999) - le titre français est Appartenance et identité -, le Canada s'est d'abord intéressé aux industries créatives pour leur apport à la culture du pays plutôt qu'à son économie. Toutefois, le rapport ne fournit pas de définition précise de ce qu'est une industrie culturelle ou créative, et les recommandations destinées à assurer sa mise en œuvre s'éloignent des préoccupations culturelles pour se tourner vers des aspects économiques.

En 1997, on note la publication du rapport Préparer le Canada au monde numérique (Industrie Canada, 1997), dans lequel il est recommandé que les entreprises multimédias bénéficient d'un crédit d'impôt. La mesure, qui au Québec porte aujourd'hui le nom de Crédit d'impôt remboursable pour la production de titres multimédias, a eu, comme nous allons le voir, un effet certain sur le secteur montréalais. On doit aussi signaler la création en 1998 du Fonds multimédia à Téléfilm Canada, qui deviendra en 2001 le Fonds des médias du Canada, et qui a pour but de soutenir la production, la distribution et la mise en marché des produits multimédias canadiens (Jeannotte, 2006). Notons enfin l'adoption, au Québec, d'une politique territoriale cherchant à favoriser l'implantation d'entreprises multimédias dans un même immeuble ou un même secteur, par des subventions conditionnelles à la localisation: ce sont les programmes ayant donné naissance à la Cité du multimédia et aux Centres de développement des technologies de l'information (Meloche et Hammouda, 2017). En résumé, la créativité semble avant tout passer par des incitatifs économiques, l'environnement socioculturel se trouvant réduit à une mécanique comptable.

L'installation progressive d'Ubisoft à Montréal à partir de 1997 est une concrétisation de ces politiques, et a laissé une trace durable sur l'organisation des entreprises de jeux vidéo au Québec. Même s'il y avait déjà un terreau fertile à Montréal à l'arrivée de l'entreprise française, c'est bien l'implantation de cette dernière, qui compte aujourd'hui plus de 3000 employés au Québec, qui a marqué le début d'une croissance importante. C'est en effet pour son arrivée que la mesure fiscale sur la production de titres multimédias a été étendue aux entreprises étrangères. «La stratégie du gouvernement est donc passée d'une mesure visant d'abord à stimuler la créativité et l'entrepreneuriat local à une politique visant à stimuler les investissements directs étrangers dans le domaine du jeu vidéo » (Meloche et Hammouda, 2017, p. 6). La créativité est instrumentalisée, mise au service d'une logique avant tout économique. Meloche et Hammouda montrent que le crédit d'impôt si généreux a surtout eu pour but de maintenir la présence de grandes entreprises 
(Ubisoft, Warner Bros, Eidos et Electronic Arts) au Québec, et que les petites et les moyennes entreprises ont dû se battre pour avoir des miettes du gâteau : si elles sont désormais admissibles aux crédits d'impôt et à certaines aides comme celle du Fonds des médias du Canada, ce n'était pas le cas au départ (Meloche et Hammouda, 2017, p. 43). Cette domination des grandes entreprises a tendance à favoriser avant tout la rentabilité. Or, ce serait généralement les petits studios indépendants qui seraient les plus créatifs (Tschang, 2007). Avec ses nombreux grands studios, l'industrie vidéoludique montréalaise ne serait donc pas aussi créative qu'on pourrait le croire.

Ces préoccupations sont partagées par la Guilde des développeurs de jeux vidéo indépendants du Québec, organisme fondé en 2016 et qui, en 2020, fusionnera avec Alliance numérique sous le nom de Guilde du jeu vidéo du Québec. À l'origine, la Guilde regroupait des créateurs de jeux vidéo et des entrepreneurs des domaines connexes établis au Québec ; elle comptait alors plus de 75 studios indépendants (La Guilde, 2016). La Guilde du jeu vidéo du Québec regroupe désormais plus de 200 entreprises liées au jeu vidéo au Québec.

Dès 2016, la Guilde s'est positionnée comme la défenseuse d'une vision culturelle du jeu vidéo, et non pas seulement comme un regroupement économique stratégique. Dans leur Mémoire présenté au ministère de la Culture et des Communications dans le cadre du renouvellement de la politique culturelle québécoise, les représentants de la Guilde insistent sur le fait que le jeu vidéo n'est pas uniquement un objet économique, mais bien un objet culturel (Malouin, Arsenault, Dagenais et Noury, 2016, p. 4). Si cet argument est nécessaire, c'est parce que, comme Meloche et Hammouda, les membres de la Guilde constatent que la politique gouvernementale a favorisé l'économie au détriment de la culture. L'innovation technologique est reconnue, tandis que l'innovation culturelle et artistique demeure peu mise de l'avant.

Si l'on compare l'argumentaire de la Guilde et celui du rapport de Meloche et Hammouda, on constate que les deux groupes s'accordent sur l'importance du développement d'une industrie locale créative : «Seule la capacité des territoires à créer et à valoriser une main-d'œuvre et un capital créatifs innovants et différenciés leur permettra de demeurer compétitifs (Malouin et al., 2016, p. 45) ». Et si l'on revient à l'origine de la notion d'industries créatives présentée plus haut, c'est aussi la créativité qui permet à un secteur de demeurer compétitif : " Les politiques axées sur les salaires bon marché (ou subventionnés) seront toujours exposées aux risques de délocalisation vers des pays où les salaires sont encore plus faibles (ou encore plus subventionnés) » (Meloche et Hammouda, 2017, p. 45).

Il y a donc une contradiction dans l'approche gouvernementale adoptée. Si c'est la créativité qui doit être soutenue, alors les mesures ne sont pas cohérentes. Les politiques semblent avoir perdu leur objectif de vue et les études suggèrent que le secteur n'est peut-être pas aussi créatif que l'on voudrait bien le croire. De plus, ces politiques ont aussi une influence sur la définition de ce que constitue un jeu vidéo, et c'est de cet aspect que nous allons désormais traiter.

\section{Le produit : les jeux}

Glăveanu souligne qu'il est nécessaire de s'intéresser aux interactions entre les différentes

dimensions de la créativité. Or, la création de nouvelles propriétés intellectuelles (product) entre dans la définition d'industrie créative. Dans notre cas, ces propriétés sont des jeux, ce sont les 
« produits » du modèle des $4 \mathrm{P}$, lesquels sont souvent réduits à des chiffres, ou à des best-sellers. Mais, comme le montrent les travaux de Gaëtan Tremblay, l'analyse de la créativité d'un produit ne peut se faire seulement par la macroéconomie (Tremblay, 2008). Les études quantitatives ne permettent pas toujours de bien comprendre la nature des produits ni ce qui constitue leur caractère créatif. Nous proposons dans cette seconde partie de confronter les travaux issus des études organisationnelles et des études de l'économie avec ceux provenant des études du jeu. Après avoir défini ce qu'est un jeu créatif, nous verrons les limites de cette définition pour le cas québécois.

\section{Qu'est-ce qu'un jeu vidéo créatif?}

En 2017, la plateforme en ligne Steam a publié 7672 jeux vidéo (Kuchera, 2018). Cette plateforme vend des jeux pour ordinateur personnel uniquement. Si l'on ajoute les jeux mobiles, les jeux sur console et les jeux pour navigateur Web, le nombre de jeux produits chaque année est énorme, et augmente d'ailleurs exponentiellement. Au Canada, cette année-là seulement, les studios ont mené à bien plus de 2000 projets de jeux vidéo : une hausse de $67 \%$ par rapport à 2015 (Entertainment Software Association of Canada, 2017). Comment déterminer ce qui relie cette multitude d'œuvres? Et lesquelles peut-on considérer comme des jeux créatifs ?

Construisant un discours sur le jeu, il semble que l'on doive définir cet objet. Il s'agit toutefois d'une entreprise malaisée (Stenros, 2016, p. 17). Dans les études de la créativité, la définition standard d'un produit créatif s'appuie sur deux critères : " Creativity requires both originality and effectiveness » (Runco et Jaeger, 2012, p. 92). L'originalité, appelée aussi nouveauté, implique quelque chose que les autres produits n'ont pas; la notion ne devrait donc pas se trouver dans la définition originelle du produit. Concernant l'efficacité (ou l'un des autres termes employés au fil du temps, comme usefulness, utility ou value), Runco note qu'il s'agit encore là d'une construction reliée à l'économie : "Effectiveness may take the form of value. This label is quite clear in the economic research on creativity; it describes how original and valuable products and ideas depend on the current market» (Runco et Jaeger, 2012, p. 92). Un jeu «créatif» serait original... et vendeur.

\section{Une jouabilité originale et de bonnes ventes}

Lorsque l'on se tourne vers les définitions des instances gouvernementales économiques et financières, les définitions du jeu vidéo sont très larges. Le jeu vidéo est généralement englobé dans les œuvres multimédias ou les logiciels informatiques. Ainsi, pour Investissement Québec, le crédit d'impôt sur les titres multimédias s'applique à toute œuvre multimédia :

Les titres doivent être édités sur un support électronique, être régis par un logiciel permettant l'interactivité et comporter, dans un volume appréciable, trois des quatre éléments suivants : texte, son, images fixes ou images animées. Il faut noter que les images vidéo ne peuvent compter pour plus d'une composante média.

Investissement Québec (2016)

Ici, le point intéressant à noter est la notion d'interactivité. Si ce terme a été repris dans les études consacrées à la narratologie et aux jeux vidéo, un autre terme pour parler de l'échange entre le jeu et le joueur se retrouve aujourd'hui dans les discours sur la créativité : "jouabilité » (gameplay, en anglais). Il est difficile de retracer l'origine de ce concept. La définition donnée par Salen et 
Zimmerman, dans leur fameux livre Rules of Play Game Design Fundamentals, revient régulièrement : "Game play is the formalized interaction that occurs when players follow the rules of a game and experience its system through play » (Salen et Zimmerman, 2003, p. 303). La jouabilité est alors liée à l'expérience du joueur.

Cependant, les contours du concept de jouabilité sont encore flous dans les études sur la créativité. Les travaux en management du professeur Ted Tschang se démarquent, car ils proposent une définition précise d'un jeu vidéo créatif. Sont retenus trois critères pour déterminer si un jeu est innovant, créatif : la jouabilité, le style visuel et l'histoire (Tschang, 2007, p. 991). Mais cela témoigne d'une vision réductrice de la jouabilité : elle se trouve isolée de l'aspect visuel et de l'histoire, alors que ces éléments sont généralement vus comme un tout. En fait, Tschang vide le concept de son sens : la jouabilité n'est plus l'expérience du joueur, mais une sorte de résidu qui persiste lorsqu'on fait abstraction des graphismes et de l'histoire.

De plus, selon Tshang, un jeu utilisant une licence préexistante, ou qui est la suite d'un autre jeu, ne constitue pas une innovation. Concernant l'efficacité d'un jeu, la question est tout aussi épineuse. Faut-il se baser sur le succès critique? Sur les ventes? Dans son étude, Tschang a tranché : puisqu'il s'agit d'une industrie, il s'appuie sur les ventes. Selon lui, un quart seulement des jeux vidéo produits en 2002 seraient de nouvelles propriétés intellectuelles, et seuls deux des vingt-cinq jeux les plus vendus seraient innovants. Il est vrai qu'en 2002, la plateforme de vente en ligne Steam n'existait pas encore, et que les jeux se présentaient principalement sur des supports pourvus d'une forme physique. En 2020, il est toutefois bien difficile de réaliser une analyse similaire à celle de Tschang.

En 2018, lors de «Montréal, capitale du jeu vidéo », conférence annuelle de Ludos à HEC Montréal, Louis-Félix Cauchon, alors président de la Guilde, a rappelé qu'une quantité très élevée d'œuvres est publiée chaque jour. Il est désormais ardu de connaitre l'état exact de la créativité dans les produits vidéoludiques tant il en existe, en particulier sur Internet, conçus par des compagnies indépendantes. Avec environ 450 studios de 25 personnes ou moins, le Canada est d'ailleurs un bon représentant du jeu vidéo indépendant, et le Québec en est même un fer de lance.

Si on applique les critères de Tschang, les titres «créatifs » produits au Québec sont bien peu nombreux. Dans la liste Wikipédia ${ }^{1}$ des cinquante jeux plus vendus, aucun n'a été créé à Montréal sans appartenir à une franchise (Wikipedia, 2018b) - il faut atteindre plus de 15 millions de copies vendues par titre pour espérer entrer dans ce classement! La plupart des grandes entreprises utilisent des licences (Eidos, Warner Brothers). On note tout de même que, avec la franchise Assassin's Creed, Ubisoft Montréal atteint les 140 millions de copies vendues (Wikipedia, 2018a).

Si l'on se tourne vers les propriétés intellectuelles des petits studios indépendants, c'est généralement le volume des ventes qui est trop restreint pour que les jeux soient considérés comme « efficaces ». On peut tout de même citer des jeux indépendants développés à Montréal qui se sont très bien vendus : Fez (Polytron Corporation, 2012) avec plus de un million d'exemplaires, ou le premier jeu de la franchise Outlast (Red Barrels, 2013) avec quatre millions de copies écoulées. Avec une définition aussi axée sur les ventes, il est malaisé pour les petits studios de tirer leur épingle du jeu. De plus, les nouveaux modèles d'affaires, dans lesquels le jeu est gratuit mais comporte des achats intégrés, rendent l'estimation des revenus compliquée. Il est aussi difficile de 
savoir ce qui constitue de bonnes ventes pour une compagnie indépendante de quelques dizaines d'employés.

Dans cette vision de la créativité centrée sur le produit, le secteur montréalais semble engagé dans une impasse. C'est ce que soulignent plusieurs études en économie et en gestion, où le ton est assez alarmiste. En effet, un phénomène particulier de «faible innovation» rongerait les entreprises. Hotho et Champion distinguent l'innovation incrémentale (ou exploitative, pour les petits changements) et l'innovation radicale (ou explorative, avec la création de produits complètement nouveaux) (Hotho et Champion, 2011). La présence d'un régime d'innovation incrémentale n'est pas considérée comme problématique par les études en gestion. Ce qui l'est, c'est l'absence du régime d'innovation radicale, régime nécessaire à la croissance et au maintien d'une entreprise (Le Masson et al., 2006): "The literature confirms that ignoring the innovation challenge will inevitably result in business failure» (Hotho et Champion, 2011, p. 35). C'est cette absence d'innovation qui est pointée dans l'industrie vidéoludique : certaines compagnies ont d'énormes difficultés à créer de nouvelles propriétés intellectuelles.

Néanmoins, si l'on observe l'évolution des jeux vidéo dans le temps, on remarque que l'innovation radicale n'est pas nécessairement ce qui caractérise ce secteur. L'innovation incrémentale existe dès la sortie de $P O N G$, en 1972. Atari ne possédant pas de brevet, des dizaines de clones vont fleurir. Il en sera de même pour DOOM (id Software, 1993), qui sera copié à loisir (Arsenault, 2011). Ce phénomène du clonage est aujourd'hui reconnu par l'industrie et la recherche (Phillips, 2015). S'appuyant sur le modèle de l'évolution des genres d'Alastair Fowler, Arseneault montre que dans les faits, le phénomène d'innovation incrémentale et de clonage est même une composante importante de l'établissement d'un genre.

À Montréal, une compagnie comme Ludia, n'hésite pas à produire des jeux dont les systèmes de règles sont des classiques d'un genre (en particulier des jeux de casino) en ne modifiant que leur thème. Dernièrement, la compagnie a publié un jeu mobile, Jurassic World Alive (2018), fortement inspiré de Pokémon Go (Niantic, 2016), mais dont le thème est repris de la série cinématographique Jurassic Park (1993-2018). Les quelques modifications apportées font que l'on peut parler de ce jeu comme d'une innovation incrémentale qui, s'inspirant du travail de Niantic, vient consacrer un genre : le jeu en réalité augmentée.

Cette réflexion sur les critères qui permettent de classer un jeu comme étant créatif ou non met en perspective les analyses qui ont pu être faites par les chercheurs en économie et en gestion. La définition d'un produit créatif devrait donc probablement être revue (Glăveanu, 2013). Ainsi, valoriser l'originalité plutôt que l'efficacité est probablement nécessaire pour arriver à des politiques qui correspondent au contexte de Montréal et de sa myriade de petits studios indépendants.

Après avoir montré que l'industrie du jeu vidéo est davantage bon marché que créative, nous venons de constater à travers l'étude des produits que les jeux vidéo sont loin d'être toujours créatifs, si l'on s'en remet aux définitions actuelles. La créativité serait-elle l'apanage des individus? 


\section{Les personnes et les processus : un manque de données}

Une quantité impressionnante de grandes icônes du domaine du jeu vidéo, comme Assassin's Creed (77 millions de copies vendues), ou Far Cry (20 millions de copies vendues), furent développées au Québec. Ces monuments n'auraient probablement jamais eu l'impact commercial qu'on leur connait si ce n'était de la présence chez nous de géants du domaine du jeu vidéo. Mais il ne faut pas oublier que c'est sur nos créateurs et créatrices que ces multinationales ont misé pour assurer leur croissance et leur survie. Et c'est en cela que les membres de La Guilde fondent leur conviction et leur ambition.

Malouin, Arsenault, Dagenais et Noury, p. 8 (2016)

Comme le rappelle ici la Guilde, derrière chaque entreprise et derrière chaque œuvre, il y a des créateurs et des créatrices. Dans le modèle originel des $4 P^{\prime}$ 's, la créativité repose sur les qualités d'une personne et sur ses attributs. Encore une fois, cette vision tend à être dépassée : l'individu s'inscrit aussi dans un contexte et est un acteur au sein d'un groupe (Glăveanu, 2013). Les employés de l'industrie vidéoludique tendent à se considérer comme créatifs ou innovants, ces adjectifs étant ici synonymes (Kultima et Alha, 2010). Ils font dès lors partie de ce que Richard Florida a nommé la classe créative. Florida, selon qui la créativité est désormais le moteur de l'économie, définit ladite classe créative comme un ensemble d'individus qui participent à la création de nouveaux contenus pertinents (Florida, 2014). Il y inclut entre autres les ingénieurs, les chercheurs et les professeurs d'université, les poètes, les romanciers, les artistes, les acteurs, les designers et les architectes. Il s'agit donc d'un groupe très diversifié. Florida évalue qu'un tiers des travailleurs de Montréal font partie de cette classe créative, et il affirme que le secteur du jeu vidéo en fournit un exemple marquant. Mais ces différents acteurs (people) ne sont pas nécessairement bien distingués et la nature de leurs actions (process) reste inégalement documentée.

\section{Production ou création?}

La création d'un jeu vidéo demande le concours de nombreuses professions, qui y apportent chacune une forme de créativité. Selon Bethke (2003), une équipe de développement de jeux vidéo dans un studio est composée de professions reliées au design, à la programmation, à l'art (artistes 2D, 3D, etc.), à l'audio, ainsi qu'au management, à l'assurance qualité (testeurs) et à la commercialisation. Les recherches s'accordent à dire que toute l'équipe contribue au caractère créatif d'un jeu et que toutes les professions apportent des éléments « innovants » (sur le plan de la jouabilité, mais aussi de la technologie, etc.).

Cependant, des tensions apparaissent pour savoir quelles idées seront adoptées, et ce ne sont pas nécessairement les professions dites créatives qui ont le dernier mot. Ainsi, on note que les éditeurs et les producteurs peuvent avoir un poids important dans la balance.

As one studio head noted, « the publishers are reading one another's hype and telling you that gamers (i.e., consumers) don't want X, Y, and Z. That's what interests me about creativity how it's completely dominated by people who are not creative. It's completely dominated by the business people $»$. 
F. Tschang, p. 993 (2007)

Cette vision est confirmée par les travaux d'Annakaisa Kultima et Kati Alha sur l'industrie mondiale du jeu vidéo. Dans leur étude réalisée auprès de designers de jeu interrogés à l'occasion de conférences professionnelles, les résultats indiquent que les éditeurs sont vus comme jouant un rôle dans l'aspect innovant d'un jeu alors qu'ils ne sont pas considérés comme créatifs. Un participant déclare : "If you go to these big publishers, they all think they are innovating. Are they? Probably not. » (Kultima et Alha, 2010, p. 3). Cette opposition entre gestion et créativité a été soulignée par des chercheurs québécois, qui en ont rapporté l'existence tant chez les développeurs indépendants qu'au sein de grandes compagnies (Pineault, 2015; Whitson, Simon et Parker, 2018).

Ce pouvoir des producteurs et des éditeurs vient peut-être du fait qu'ils dirigent les processus créatifs, et que la littérature en gestion, sous l'étiquette d'organizational creativity, a modélisé lesdits processus selon différents grands courants : le Creative Problem Solving de Osborn, le design thinking de Tim Cook ou encore la theory of inventive problem solving (Puccio et Cabra, 2010). Dans le développement de jeux vidéo, c'est la méthode Agile qui semble la plus utilisée (Politowski, Fontoura, Petrillo et Guéhéneuc, 2016). Il s'agit en fait d'un ensemble de méthodes de gestion ou de pratiques censées encourager la créativité en s'éloignant d'une vision rationnelle et contrôlée du processus d'élaboration d'un logiciel (Hodgson et Briand, 2013). Comme le montre par exemple l'étude des entreprises de jeux vidéo du quartier Saint-Roch à Québec, les entreprises et les centres de formation de la Belle Province utilisent Agile pour gérer l'innovation (Sauvé, Briand et Hanin, 2010). Ces théories sont aussi mises à contribution pour étudier d'autres pratiques, par exemple celles à l'œuvre dans la gestion de communautés de joueurs-créateurs à Montréal (Grandadam, Simon, Marchadier et Tremblay, 2010).

\section{Le design dans la création de jeux vidéo}

Si l'apport des sciences de la gestion est indéniable, ce sont les professions liées au design qui restent majoritairement créditées pour leur apport créatif (Kultima, 2010) : design de systèmes, design narratif, ou encore design de personnages et d'environnements (appelé aussi concept art). Dans la hiérarchie des projets, le designer en chef (lead) est d'ailleurs régulièrement nommé «directeur créatif»: il se porte garant de la cohérence du projet. Dans le rapport de sir George Cox sur les industries créatives, le design est conséquemment défini comme l'activité de mise en pratique de la créativité (Schlesinger, 2007).

Alors que toute une équipe de designers sera à l'œuvre sur un projet qui requiert plusieurs centaines de personnes, on note malgré tout que l'image du designer vedette est encore présente (Chiapello, 2015). Le designer de jeu fascine, et l'on pousse sur le devant le scène un seul individu, un créateur tout-puissant : Warren Spector, Shigeru Miyamoto ou Will Wright. Montréal a ainsi son début de panthéon, avec Patrice Désilet, créateur d'Assassin's Creed. Cette insistance sur le designer de génie peut être reliée aux études sur la créativité, qui se sont longtemps concentrées sur des individus singuliers, extraordinaires, afin de définir l'essence de la créativité, qui est subséquemment vue comme un trait que certains possèdent plus que d'autres (Bilton et Leary, 2002, p. 54). Cette image du génie peut aussi être liée au fait que les designers de jeu sont souvent des autodidactes. Ainsi, lorsqu'elles interrogeaient des designers de jeux, Kultima et Alha ont noté 
que peu d'entre eux avaient étudié les jeux vidéo (Kultima et Alha, 2010, p. 4). Recruter un designer de jeu n'est d'ailleurs pas une chose facile. Il y a pénurie de talents à Montréal et le gouvernement fédéral a instauré, avec son programme Talents mondiaux (Gouvernement du Canada, 2018), une politique de recrutement visant à faire immigrer au Canada des designers de jeu chevronnés. Cette pénurie montre que malgré les nombreux programmes de formation montréalais, le bassin de travailleurs qualifiés reste restreint.

Une formation adéquate est toutefois un paramètre important pour la stabilité du secteur (Malouin et al., 2016, p. 11). Aujourd'hui, à Montréal, la plupart des baccalauréats en jeu vidéo incluent des cours de design de jeu, que ce soit à l'Université Concordia, à l'Université de Montréal, au Centre de l'Université du Québec en Abitibi-Témiscamingue, ou encore à l'École des arts numériques, de l'animation et du design de l'Université du Québec à Chicoutimi. Ces formations font la part belle à la créativité (Bouchard, 2017; UQAT, 2018), et promettent de former des "professionnels créatifs » ou de « développer la créativité des étudiants », qui est vue comme un trait individuel nécessaire aux futurs designers.

On pourrait penser que la créativité des designers de jeux vidéo a donc été évaluée, quantitativement ou qualitativement. Mais la littérature à ce sujet est plutôt mince. En fait, malgré les nombreuses techniques existantes, il apparait qu'il est difficile d'évaluer la créativité des employés des industries créatives, car chaque méthode comporte des lacunes, en particulier en ce qui concerne le contexte dans lequel évoluent les individus (Chen et Kaufmann, 2008). Il ne semble y avoir aucune preuve ni aucune description permettant de comprendre en quoi les designers de jeu sont créatifs.

De même, en ce qui a trait aux processus, la littérature en design de jeu est peu fournie. Dans le modèle originel des $4 \mathrm{P}$, la dimension des processus se rapporte généralement uniquement aux mécanismes cognitifs d'un individu. C'est pourquoi Glăveanu préfère désormais utiliser le terme action pour désigner l'ensemble des activités des acteurs. Mais que ce soit comme processus cognitif ou comme activité, le fait de concevoir des jeux demeure sous-étudié comparativement, par exemple, au fait de jouer à ces jeux. En se penchant sur les modèles du design de jeu présent dans la littérature, Kuittinen et Holopainen constatent que la vision est toujours celle d'une boîte noire, d'une activité «magique » (Kuittinen et Holopainen, 2009, p. 5). Ce sont davantage les jeux en eux-mêmes qui sont analysés :

Judging from the selection of the game design literature we analysed, game design is heavily governed by the object of the design, games. Although this may seem like an overly obvious statement, it carries with itself the connotation that the activity called design, is left to too little attention.

Jussi Kuittinen et Jussi Holopainen, p. 7 (2009)

La plupart du temps, les travaux en design de jeu s'appuient sur un modèle du design vague et implicite. Kuittinen et Holopainen notent que fréquemment, les modèles du processus de design proposent des étapes dont l'une est elle-même récursivement nommée design (Kuittinen et Holopainen, 2009). Ils ajoutent que le design est souvent cantonné à l'étape de la pré-production, au tout début du développement d'un jeu, et que les différentes étapes et les actions des designers 
restent mal définies. Djaouti et Alvarez arrivent à une conclusion similaire : "Cette dernière catégorie de modèles [sur les processus de design] nous amène à réaliser qu'il ne semble pas exister une "série d'étapes universelles" permettant de définir le processus de conception d'un jeu » (Djaouti, Alvarez et Jessel, 2010, p. 27).

La compréhension de ce que font les designers reste d'ailleurs floue pour les designers eux-mêmes (Kultima, 2010). De plus, contrairement aux gestionnaires, les designers de jeux vidéo ne sont pas rompus aux techniques de créativité (Kultima et Alha, 2010). Ils en connaissent certaines de nom, comme le remue-méninges (brainstorming) et Agile, mais ne savent pas nécessairement bien les utiliser (Kultima, 2010). Dans la littérature en design, les concepts de créativité et d'innovation sont utilisés de façon interchangeable (Hernández et al., 2018). Les chercheurs paraissent alors avoir du mal à trouver des modèles permettant de décrire ce qui se déroule dans l'activité de design, ce qui empêche de discerner les actions créatives au sein de l'entreprise.

Ce manque de modèle a posé problème au sein de l'industrie, et on remarque que les designers, forts de leur expérience, se forgent leurs propres approches. (Kultima, 2010). Ainsi, Ubisoft a développé une méthode, le rational design (McEntee, 2012). Si cette vision demeure centrée sur le jeu plus que sur le processus, elle témoigne de la volonté de l'entreprise de mieux comprendre le design. De plus, les anciens employés d'Ubisoft peuvent propager cette vision. Par exemple, Alexis Jolis-Desautels, qui a fondé son entreprise de jeux, Lucky Hammers, continue d'utiliser cette approche, et l'enseigne au Campus ADN, un centre de formation en arts et en divertissement numérique situé à Montréal. Le rational design est progressivement en train de pénétrer l'industrie québécoise. Or, cette méthode, comme son nom l'indique, s'attache à rationaliser le processus, c'est-à-dire qu'elle incite à se focaliser sur l'aspect de la productivité et de l'efficacité, généralement au détriment de l'originalité et donc de la créativité (Tschang, 2007).

En résumé de cette dernière partie, nous avons suivi les recommandations de Glăveanu et étudié ensemble les acteurs et leurs activités, ce qui permet de constater que certains aspects de la créativité vidéoludique restent méconnus : les activités de design, jugées créatives, sont encore mal comprises. Cette vision floue du processus de design de jeu contraste avec celle, beaucoup plus développée, des processus créatifs en gestion. Cela donne une place prépondérante aux processus de gestion, mais ceux-ci ne couvrent pas entièrement la création; de plus, leur pertinence pour la création de jeux vidéo reste à prouver. Peu de liens sont faits entre design et gestion, et certains se demandent même si Agile encourage réellement la créativité (Hodgson et Briand, 2013). Par ailleurs, dans les travaux en gestion, certaines activités de design, par exemple le design d'un personnage ou d'un environnement, sont parfois amalgamées avec des aspects techniques comme la modélisation du personnage ou de l'environnement dans le logiciel en 3D (Parmentier et Mangematin, 2009), ce qui rend l'analyse moins pertinente.

Devant ce manque de liens entre les disciplines, il demeure difficile d'établir que les employés des entreprises de jeux vidéo québécoises et leur processus sont particulièrement créatifs, et l'on peut se demander si le discours sur la classe créative n'est pas que de la poudre aux yeux pour mieux exploiter les individus, comme le dénoncent certains depuis longtemps (Dyer-Witheford et De Peuter, 2006; ea_spouse, 2004). De plus, dans un contexte de délocalisation et de remplacement de certains métiers, il serait bon de savoir quelle est la place du design et de la créativité chez les employés des entreprises québécoises. Plutôt que de blâmer le marketing ou le management quand 
se produisent des échecs commerciaux, il faudrait pouvoir comprendre comment s'est déroulé le processus de création. Cela pourra donner plus de pouvoir aux designers : celui d'améliorer leur façon de faire, de s'adapter et d'assumer pleinement leur rôle de « directeur créatif ».

\section{Conclusion}

Cet article proposait de nuancer la vision positive de la créativité dans l'industrie vidéoludique au Québec, et surtout à Montréal, en interrogeant les limites du concept. En utilisant la version de Glăveanu du modèle des $4 \mathrm{P}$, la notion de créativité a été abordée à partir de l'environnement des industries créatives, la perspective se resserrant progressivement sur le produit pour finalement aboutir à l'activité des designers de jeu. Les conclusions au sujet de l'environnement montrent que, plus que la créativité, c'est la main-d'œuvre bon marché qui fait de Montréal une ville attrayante. Les réflexions sur les jeux dévoilent que, loin de créer sans cesse des innovations, les compagnies créent souvent des œuvres appartenant à un genre, ou pire, produisent des clones. Lorsque l'on se penche sur la question des employés, on constate la place centrale qu'occupent les designers dans le processus créatif, bien qu'on ne puisse pas précisément décrire en quoi consiste leur créativité ni ce qu'il en est de leur processus créatif. Finalement, lorsque l'on rapproche les quatre dimensions, la province de Québec et la ville de Montréal en particulier apparaissent peu créatives.

L'industrie vidéoludique québécoise ressemble à un colosse aux pieds d'argile : énorme en termes de revenus, mais fragile lorsque l'on aborde ce sur quoi elle repose, la créativité. Faudrait-il alors repousser l'idée même de créativité ? C'est ce que proposait récemment Florida (2017) : après avoir présenté Montréal comme un modèle à suivre au Canada, il affirme désormais qu'une économie de la créativité renforce les inégalités. Cependant, il ne faudrait pas jeter le bébé avec l'eau du bain. D'autant plus que l'aura de la créativité ne semble pas près de s'éteindre, comme en témoignent les politiques culturelles et économiques au Québec ou le développement des formations en jeu vidéo. Une fois repris dans les sphères politico-économiques, un concept comme celui d'industrie créative a un impact sur tous les acteurs du secteur. Une vision critique et nuancée est dès lors de mise. Les échecs dans l'industrie ont des répercussions concrètes : licenciements, calcul des risques se soldant par des investissements frileux, uniformisation de la culture vidéoludique, etc.

Il est plutôt nécessaire de remettre en question la théorisation de la créativité. Nous avons vu que, pris sous différents angles selon les discours, le concept présente finalement tellement de limites qu'il n'a plus aucun sens. Par exemple, les définitions actuelles de ce qu'est un jeu créatif sont loin de nous livrer une vision claire de la production québécoise. Au lieu d'ignorer un tel flou, il parait plus judicieux d'approfondir nos fondements théoriques.

Le modèle socioculturel de Glăveanu permet de proposer des pistes pour améliorer la situation. Sur le plan des personnes et des processus, notre analyse fait ressortir la précarité et le manque de reconnaissance des professions dites créatives. Si les emplois liés au design (design du jeu, des personnages, des environnements, etc.) sont vus comme centraux pour l'économie, ils demeurent tout de même peu connus, mal définis. On note le manque d'études de terrain, le manque de recherches portant sur les pratiques des créateurs. Effectuer des recherches en collaboration avec des praticiens constitue donc une voie prometteuse de développement des connaissances, et plusieurs chercheurs au Québec se sont engagés dans cette voie. On peut penser par exemple aux 
études de studio lancées par Jennifer Whitson à Concordia, aux recherches en cours de Dave Hawey à l'École des arts numériques, de l'animation et du design de l'UQAC, à celles de Gabrielle Trépanier-Jobin et de Maude Bonenfant à l'UQAM.

Sur un plan plus théorique, l'évolution du modèle des $4 \mathrm{P}$ est un signe de la nécessité de repenser la vision dominante de la créativité. Cette vision définit les produits créatifs sans tenir compte de leur contexte et ne met pas suffisamment l'accent sur les actions des acteurs. Au contraire, l'étude de la situation de l'industrie vidéoludique québécoise montre que le concept de créativité mérite que l'on continue de l'explorer. Des liens entre des disciplines comme la gestion et le design demandent à être construits (Johansson-Sköldberg, Woodilla et Çetinkaya, 2013). Nous avons aussi contribué à ce courant qui veut que la recherche utilise des modèles issus des disciplines du design (architecture, urbanisme, design industriel, design d'intérieur) pour les appliquer dans les études du jeu (Chiapello, 2017). Il s'agit d'une voie prometteuse : elle offre un cadre conceptuel riche et peut éviter des écueils, notamment l'excès de rationalité.

Enfin, en défendant une vision transdisciplinaire (Létourneau, 2008; Montuori, 2013), nous espérons participer au développement de la vision constructiviste de la créativité. Un nouveau laboratoire de recherche, PRAXIS, a ainsi été créé en 2018 à l'École des arts numériques, de l'animation et du design de l'UQAC. Il a pour vocation d'étudier les pratiques dans l'écosystème vidéoludique québécois et de mettre en valeur les savoirs des professionnels, des artistes aux designers en passant par les programmeurs. L'approche y est constructiviste pragmatiste, et veut dépasser les frontières disciplinaires. Cette transdisciplinarité demandera toutefois une réflexion épistémologique profonde afin d'offrir des analyses cohérentes de la créativité vidéoludique, qui, nous l'espérons, pourront mieux saisir les activités qui se déroulent à Montréal et au Québec.

\section{Remerciements}

Je souhaite remercier Bernard Perron pour son soutien et ses corrections tout au long de l'élaboration de cet article, Anouk Bélanger pour ses commentaires avisés et Odette Provost pour sa révision linguistique. Cette recherche a été effectuée dans le cadre de ma thèse de doctorat en aménagement, dirigée par Rabah Bousbaci et financée par une bourse du Fonds de recherche du Québec - société et culture.

\section{Références}

Alliance numérique. (2014). Perpétuons le miracle québécois: l'avenir de l'industrie du jeu vidéo au Québec. En ligne : $\quad$ http://alliancenumerique.com/wpcontent/uploads/2016/05/Mémoire_AN-commission-Godbout-2014.pdf.

Anadón, M. (2006). La recherche dite «qualitative»: de la dynamique de son évolution aux acquis indéniables et aux questionnements présents. In Recherches qualitatives, 26(1), 5-31.

Arsenault, D. (2011). Des typologies mécaniques à l'expérience esthétique : fonctions et mutations du genre dans le jeu vidéo. (Ph.D. thèse en études cinématographiques). Université de Montréal, Montréal.

Benessaieh, K. (2017). Fin de partie pour le studio Hibernum. In La Presse, 17 juillet. En ligne : https://www.lapresse.ca/techno/jeux-video/201708/17/01-5125239-fin-de-partie-pour-le- 
studio-hibernum.php.

Bethke, E. (2003). Game development and production. Plano, Texas: Wordware Publishing Inc.

Bilton, C., et Leary, R. (2002). What can managers do for creativity? Brokering creativity in the creative industries. In International Journal of Cultural Policy, 8(1), 49-64.

Bouchard, S. (2017). L'UQAC lance un programme en création de jeux vidéo. In Le Journal de Québec, 11 décembre. En ligne : https://www.journaldequebec.com/2017/12/11/luqaclance-un-programme-en-creation-de-jeux-video.

Bousbaci, R. (2008). "Models of Man" in Design Thinking: The "Bounded Rationality" Episode. In Design Issues, 24(4), 38-52. doi:10.1162/desi.2008.24.4.38

Chambre de commerce du Montréal métropolitain. (2018). Industries créatives : réussir dans un environnement en mutation rapide. En ligne : https://www.ccmm.ca/fr/publications/etude/collaboration-art-affaires/industries-creatives-reussir-dans-un-environnement-en-mutation-rapide/.

Chen, M. H., et Kaufmann, G. (2008). Employee creativity and R\&D: A critical review. In Creativity and Innovation Management, 17(1), 71-76.

Chiapello, L. (2015). Le designer de jeux vidéo comme agent d'évolution à l'ère du jeu casual. InSciences du jeu, 4, En ligne : https://sdj.revues.org/474.

Chiapello, L. (2017). Epistemological Underpinnings in Game Design Research. In P. Lankoski et J. Holopainen (dirs.), Game Design Research: An Introduction to Theory \& Practice (p. 15-33). Pittsburgh, PA,: Carnegie Mellon University ETC Press.

Chiapello, L. (2019). Le pragmatisme comme épistémologie pour le design de jeux Enquête sur la créativité et le processus de design. (Thèse de doctorat Thèse de doctorat). Université de Montréal, Montréal.

Cohendet, P., Llerena, P., et Simon, L. (2012). The Routinization of Creativity : Lessons from the Case of a video-game Creative Powerhouse. En ligne : file:///C:/Users/perro/AppData/Local/Temp/The_Routinization_of_Creativity_Lessons_fr om the C.pdf.

Cohendet, P., et Simon, L. (2007). Playing across the playground: paradoxes of knowledge creation in the videogame firm. In Journal of Organizational Behavior, 28(5), 587-605.

Communauté des jeux vidéo du Québec. (2020). Jeux vidéo du Québec. En ligne: http://www.gamesfromquebec.com/fr/.

Djaouti, D., Alvarez, J., et Jessel, J.-P. (2010). Concevoir l'interactivité ludique: une vue d'ensemble des méthodologies de 'Game Design'. Paper presented at the Ludovia 2010 Ariège-Pyrénées.

Drake, P. (2013). Policy or Practice? Deconstructing the Creative Industries. In P. Szczepanik et P. Vonderau (dirs.), Behind the screen: inside european production cultures (p. 221-236). 
New York: Palgrave Macmillan.

Dyer-Witheford, N., et De Peuter, G. (2006). "EA Spouse" and the crisis of video game labour: Enjoyment, exclusion, exploitation, exodus. In Canadian Journal of Communication, 31(2006), 599-617.

Dyer-Witheford, N., et Sharman, Z. (2005). The political economy of Canada's video and computer game industry. In Canadian Journal of Communication, 30(2), 187-210.

ea_spouse. (2004). EA: The Human Story. En ligne : https://ea-spouse.livejournal.com

Entertainment Software Association of Canada. (2017). Canada's Video Game Industry in 2017. En ligne : http://theesa.ca/wp-content/uploads/2017/10/ESAC2017_Booklet_French05 Digital.pdf.

Flew, T. (2002). Beyond ad hocery: Defining creative industries. Paper presented at the Cultural Sites, Cultural Theory, Cultural Policy, The Second International Conference on Cultural Policy Research, Te Papa, Wellington, New Zealand.

Florida, R. (2014). The rise of the creative class--revisited: Revised and expanded. New York: Basic Books.

Florida, R. (2017). The new urban crisis: How our cities are increasing inequality, deepening segregation, and failing the middle class-And what we can do about it. New York: Basic Books.

Glăveanu, V. P. (2013). Rewriting the language of creativity: The Five A's framework. In Review of General Psychology, 17(1), 69.

Glăveanu, V. P. (2014). The psychology of creativity: A critical reading. In Creativity: Theories Research - Applications, 1(1), 10-32.

Glăveanu, V. P. (dir.) (2019). The Creativity Reader. New York, NY: Oxford University Press.

Glăveanu, V. P., Hanchett Hanson, M., Baer, J., Barbot, B., Clapp, E. P., Corazza, G. E., Hennessey, B., James C. Kaufman, J.C., Lebuda, I., Lubart, T. , Montuori, A., Ness, I.J., Plucker, J., Reiter-Palmon, R., Sierra Z.,Simonton, D.K., Neves-Pereira, M.S et Sternberg, R.C. (2019). Advancing Creativity Theory and Research: A Socio-cultural Manifesto. In The Journal of Creative Behavior, 1-5. doi:10.1002/jocb.395.

Gouvernement du Canada. (2018). Exigences du Programme pour le Volet des talents mondiaux. En ligne : https://www.canada.ca/fr/emploi-developpement-social/services/travailleursetrangers/talents-mondiaux/exigences.html\#cus.

Grandadam, D., Cohendet, P., et Simon, L. (2012). Places, Spaces and the Dynamics of Creativity: The Video Game Industry in Montreal. In Regional Studies, 47(10), 1701-1714. doi:10.1080/00343404.2012.699191.

Grandadam, D., Simon, L., Marchadier, J., et Tremblay, P.-O. (2010). Gérer des communautés de création: Ubisoft Montréal et les jeux vidéo. In Gestion, 35(4), 56-63. 
Gruszka, A., et Tang, M. (2017). The 4P's creativity model and its application in different fields. In Handbook of the Management of Creativity and Innovation: Theory and Practice (p. 5171). Singapore: WSPC

Hernández, R. J., Cooper, R., Tether, B., et Murphy, E. (2018). Design, the Language of Innovation: A Review of the Design Studies Literature. In She Ji, 4(3), 249-274.

Hesmondhalgh, D. (2008). Cultural and creative industries. In T. Bennett et J. Frow (dirs.), The SAGE handbook of cultural analysis (p. 553-569). London, UK: Sage Publications.

Hodgson, D., et Briand, L. (2013). Controlling the uncontrollable: 'Agile' teams and illusions of autonomy in creative work. In Work, Employment and Society, 27(2), 308-325. doi:10.1177/0950017012460315.

Hotho, S., et Champion, K. (2011). Small businesses in the new creative industries: Innovation as a people management challenge. In Management Decision, 49(1), 29-54.

Industrie Canada. (1997). Préparer le Canada au monde numérique : rapport final du Comité consultatif sur l'autoroute de l'information. En ligne : http://publications.gc.ca/site/eng/9.680195/publication.html.

Investissement Québec. (2016). PME et grandes entreprises crédits d'impôt pour production de titres multimédias. En ligne : http:/www.investquebec.com/quebec/fr/produitsfinanciers/pme-et-grandes-entreprises/credit-d-impot/production-de-titresmultimedias.html.

Jeannotte, S. (2006). Timeline of Canadian Federal Cultural Policy Milestones 1849 to 2005. Em ligne : http://socialsciences.uottawa.ca/governance/sites/socialsciences.uottawa.ca.g overnance/files/timeline.pdf.

Johansson-Sköldberg, U., Woodilla, J., et Çetinkaya, M. (2013). Design thinking: past, present and possible futures. In Creativity and Innovation Management, 22(2), 121-146.

Kuchera, B. (2018). Report: 7,672 games were released on Steam in 2017. En ligne : https://www.polygon.com/2018/1/10/16873446/steam-release-dates-2017.

Kuittinen, J., et Holopainen, J. (2009). Some notes on the nature of game design. Paper presented at the 2009 DiGRA Conference: Breaking New Ground, Brunel University, London.

Kultima, A. (2010). The organic nature of game ideation: game ideas arise from solitude and mature by bouncing. Paper presented at the International Academic Conference on the Future of Game Design and Technology, Vancouver, British Columbia, Canada.

Kultima, A., et Alha, K. (2010). “Hopefully everything I'm doing has to do with innovation”. Paper presented at the International IEEE Consumer Electronics Society's Games Innovations Conference, Hong Kong.

La Guilde des développeurs de jeux vidéo indépendants du Québec. (2016). La plus grosse coopérative de jeux vidéo indépendants au monde voit le jour au Québec. En ligne : https://www.esmtl.ca/nouvelles/article/la-plus-grosse-cooperative-de-jeux-videos-inde 
pendants-au-monde-voit-le-jour-au-quebec/.

Le Masson, P., Weil, B., et Hatchuel, A. (2006). Les processus d'innovation : conception innovante et croissance des entreprises. Paris: Lavoisier.

Létourneau, A. (2008). La transdisciplinarité considérée en général et en sciences de l'environnement. In VertigO-la revue électronique en sciences de l'environnement, 8(2), 1-9. En ligne : https://www.erudit.org/fr/revues/vertigo/2008-v8-n2vertigo2774/019961ar.pdf.

Malouin, J.-F., Arsenault, D., Dagenais, O., et Noury, M. (2016). Le rayonnement de la culture québécoise par le jeu vidéo. Mémoire présenté au ministère de la Culture et des Communications dans le cadre du renouvellement de la politique culturelle québécoise. En ligne : https:/www.mcc.gouv.qc.ca/fileadmin/documents/Politiqueculturelle/Memoires Metadonnees/La_Guilde_des_developpeurs_de_jeux_video_inde pendants_du_Quebec.pdf.

McEntee, C. (2012, October). Rayman Origins Post Mortem. In Game Developer, 19(10), 26-31.

Meloche, J.-P., et Hammouda, K. (2017). L'aide fiscale à l'industrie du jeu vidéo à MontréalQuelques éléments de réflexion. Rapport pour discussion. En ligne : https://www.cirano.qc.ca/files/publications/2017DT-01.pdf.

Metacritic. (2012). The Secret World PC. En ligne : http://www.metacritic.com/game/pc/thesecret-world

Ministerial Creative Industries Strategy Group. (2001). Creative Industries Mapping Documents. Rapport pour le Department of Culture, Media and Sport. En ligne : https://www.gov.uk/government/publications/creative-industries-mapping-documents$\underline{2001 .}$.

Montuori, A. (2013). The complexity of transdisciplinary literature reviews. In Complicity: An International Journal of Complexity and Education, 10(1/2), 45-55é

Oakley, K. (2009). The disappearing arts: Creativity and innovation after the creative industries. In International Journal of Cultural Policy, 15(4), 403-413.

Parmentier, G., et Mangematin, V. (2009). Innovation et création dans le jeu vidéo. Comment concilier exploration et exploitation? In Revue française de gestion, 191(1), 71-87. En ligne : https://www.cairn.info/revue-francaise-de-gestion-2009-1-page-71.htm.

Phillips, T. (2015). "Don't clone my indie game, bro": Informal cultures of videogame regulation in the independent sector. In Cultural Trends, 24(2), 143-153.

Pineault, Y. (2015). Le jeu vidéo à Montréal: une négociation entre création et production. In Sciences du jeu, 4, 1-17. En ligne : https://journals.openedition.org/sdj/494?lang=en.

Plucker, J. A., Beghetto, R. A., et Dow, G. T. (2004). Why isn't creativity more important to educational psychologists? Potentials, pitfalls, and future directions in creativity research. In Educational Psychologist, 39(2), 83-96. 
Politowski, C., Fontoura, L., Petrillo, F., et Guéhéneuc, Y.-G. (2016). Are the old days gone?: a survey on actual software engineering processes in video game industry. Paper presented at the 5th International Workshop on Games and Software Engineering, Austin, Texas.

Puccio, G. J., et Cabra, J. F. (2010). Organizational creativity. In J. C. Kaufman et R. J. Sternberg (dirs.), The Cambridge handbook of creativity (p. 145-173). Cambridge, MA: Cambridge University Press.

Rhodes, M. (1961). An analysis of creativity. In The Phi Delta Kappan, 42(7), 305-310.

Runco, M. A. (2004). Creativity. In Annual Review of Psychology, 55(1), 657-687. doi:10.1146/annurev.psych.55.090902.141502

Runco, M. A., et Jaeger, G. J. (2012). The standard definition of creativity. In Creativity Research Journal, 24(1), 92-96.

Salen, K., et Zimmerman, E. (2003). Rules of Play Game Design Fundamentals. Cambridge, MA: MIT Press.

Sauvé, N., Briand, L., et Hanin, F. (2010). Étude exploratoire des entreprises du savoir: le cas des développeurs de jeux vidéo du quartier Saint-Roch de Québec: Centre de recherche sur les innovations sociales.

Schlesinger, P. (2007). Creativity: from discourse to doctrine? In Screen, 48(3), 377-387.

Schlesinger, P. (2012). Expertise, politiques publiques et économie créative: le cas britannique. In Actes de la recherche en sciences sociales, 193(3), 80-95.

Schlesinger, P. (2017). The creative economy: invention of a global orthodoxy. In Innovation: The European Journal of Social Science Research, 30(1), 73-90.

Schön, D. A. (1983). The Reflective Practitioner : How Professionals Think in Action. New York: Basic Books.

Standing Committee on Canadian Heritage. (1999). A Sense of Place, a Sense of Being. The Evolving Role of the Federal Government in the Support of Culture in Canada, 9th Report of the Standing Committee on Canadian Heritage. En ligne : www.pch.gc.cal.

Stenros, J. (2016). The Game Definition Game: A Review. In Games and Culture, 1(22), 1-22.

Tremblay, G. (2008). Industries culturelles, économie créative et société de l'information. In Global Media Journal, 1 (Inaugural Issue), 65-88.

Tschang, F. T. (2007). Balancing the tensions between rationalization and creativity in the video games industry. In Organization Science, 18(6), 989-1005.

UQAT. (2018). Baccalauréat en création de jeux vidéo Profils art et design. En ligne : https://www.uqat.ca/etudes/creation-et-nouveaux-medias/baccalaureat-en-creation-dejeux-video-profils-art-et-design/.

Weber, R. (2012). Secret World designer: MMO needed to be more commercial. En ligne : 
https:/www.gamesindustry.biz/articles/2012-10-09-secret-world-designer-mmo-neededto-be-more-commercial.

Weiner, R. (2000). Creativity \& beyond cultures, values, and change. Albany, NY: State University of New York Press.

Whitson, J. R., Simon, B., et Parker, F. (2018). The Missing Producer: Rethinking indie cultural production in terms of entrepreneurship, relational labour, and sustainability. In European Journal of Cultural Studies, décembre, 1-22.

Wikipedia. (2018a). List of best-selling video game franchises. Wikipedia. En ligne : https://en.wikipedia.org/wiki/List_of best-selling_video_game_franchises.

Wikipedia. (2018b). List of best-selling video games. Wikipedia. En ligne : https://en.wikipedia.org/wiki/List_of_best-selling_video_games?oldid=559733465.

\footnotetext{
${ }^{1}$ Les compagnies de jeux vidéo ne rendent que rarement publics les chiffres de leurs ventes. La liste de Wikipédia, si elle est loin d'être parfaitement fiable, offre au moins des ordres de grandeur, ce qui donne un portrait du marché.
} 